\title{
Microscopic understanding of the photoconduction effect in graphene
}

\author{
Roland Jago, ${ }^{1, *}$ Florian Wendler, ${ }^{2}$ and Ermin Malic ${ }^{1}$ \\ ${ }^{1}$ Chalmers University of Technology, Department of Physics, SE-412 96 Gothenburg, Sweden \\ ${ }^{2}$ Institut für Theoretische Physik, Technische Universität Berlin, 10623 Berlin, Germany
}

(Received 30 May 2017; published 22 August 2017)

\begin{abstract}
We investigate the photoresponse of intrinsic graphene in an in-plane electric field. Toward that end, we employ a microscopic approach that allows us to determine the time- and momentum-resolved charge-carrier distributions as a result of the interplay between the field-induced acceleration of optically excited carriers and Coulomb- and phonon-driven carrier scattering. Calculating the generated photocurrent that is determined by the asymmetry of the carrier distribution, we reveal the microscopic foundation of the photoconduction effect in graphene. In particular, we discuss the possibility of tuning the photocurrent via externally accessible knobs, such as electric field, temperature, and substrate. Furthermore, we study the impact of Auger-induced carrier multiplication on the photocurrent in graphene.
\end{abstract}

DOI: 10.1103/PhysRevB.96.085431

In recent years, a wide variety of photodetectors based on graphene have been demonstrated showing several advantages over conventional photodetectors, such as a broad spectral range and ultrafast carrier dynamics [1,2]. However, the underlying mechanism of the photodetection is not always clear, since different mechanisms can potentially induce a photocurrent [1,3]: (i) the photoconduction effect relies on increased conductivity due to a photoenhanced carrier density, (ii) the photovoltaic effect describes the separation of photoexcited electron-hole pairs in an internal electric field generated in a $p$ - $n$ junction or a Schottky barrier, (iii) the bolometric effect allows the detection of light through a conductivity change induced by heating the sample via absorption of photons, and (iv) the photothermoelectric effect occurs if a heat gradient is created by photon absorption that is converted into a voltage difference via the Seebeck effect.

The first graphene-based photocurrents were measured in graphene-metal and metal-graphene-metal junctions [4-8], where the photovoltaic effect is important. In further studies, the photothermoelectric effect was shown to be the dominant mechanism in the case without external bias [9-12], while under bias additional contributions due to the photoconduction and the photobolometric effects arise [13-16]. This class of photodetectors is characterized by an ultrafast response, but it does not allow the detection of low intensity radiation. To detect also small numbers of photons, a high responsivity (ratio of photocurrent to incident optical power) is needed. The highest responsivities have so far been achieved in hybrid photodetectors based on the photogating effect, which is a particular example of the photoconduction effect $[17,18]$. In these detectors, the photons are absorbed in quantum dots or another graphene sheet separated by a tunnel barrier, where the created charge carriers are trapped and act as a gate changing the conductance of the graphene sheet.

To exploit the full potential of the graphene-based photodetectors, it is important to understand the carrier dynamics in graphene in an electric field under illumination, i.e., the microscopic origin of the photoconduction effect. In particular, the recently theoretically predicted [19-23] and

\footnotetext{
*roland.jago@chalmers.se
}

experimentally measured $[20,24-26]$ carrier multiplication is a promising ultrafast phenomenon since it creates additional charge carriers through internal Auger scattering.

In a recent study, we investigated the carrier dynamics of graphene in an in-plane electric field, revealing that fieldinduced Auger scattering results in a significant charge carrier density enhancement that we denote as dark carrier multiplication. We have found an asymmetric quasiequilibrium electron distribution resulting from an interplay of field-induced carrier acceleration and carrier-carrier and carrier-phonon scattering giving rise to current amplification [27,28]. In this work, we investigate the photoresponse of graphene in an in-plane electric field, where the photoconduction effect is expected to be dominant, while under the assumption of uniform illumination of a homogeneous sample the photothermoelectric effect can be ruled out. Based on a microscopic approach within the density matrix formalism [29,30], we derive graphene Bloch equations allowing us to track the photoexcited charge carriers in momentum and time, as they are accelerated by the electric field and as they scatter with one another as well as with phonons.

An electric field $\mathbf{E}$ pointing in the $-x$ direction shifts carriers in the $k_{x}$ direction in momentum space and induces a dark current [27]. Scattering processes counteract the acceleration and lead to a stationary carrier distribution $\rho_{\mathbf{k}}^{0}$ [Fig. 1(a)], which has an increased carrier density due to field-induced dark carrier multiplication [27]. To study the photocurrent dynamics, carriers are excited by a linearly polarized (in the $y$ direction) optical pulse of an exemplary energy of $0.6 \mathrm{eV}$, a temporal width of $1 \mathrm{ps}$, and a fluence of $1 \mu \mathrm{J} \mathrm{cm}$. This creates a nonequilibrium carrier distribution with high energetic electron-hole pairs [Fig. 1(b)]. These optically induced charge carriers are accelerated in the electric field and perform simultaneous Coulomb- and phonon-induced scattering processes. This gives rise to an interesting carrier and photocurrent density dynamics, which is the focus of this work.

\section{THEORETICAL APPROACH}

To microscopically model the dynamics of optically excited carriers in the presence of an electrical field, we derive 

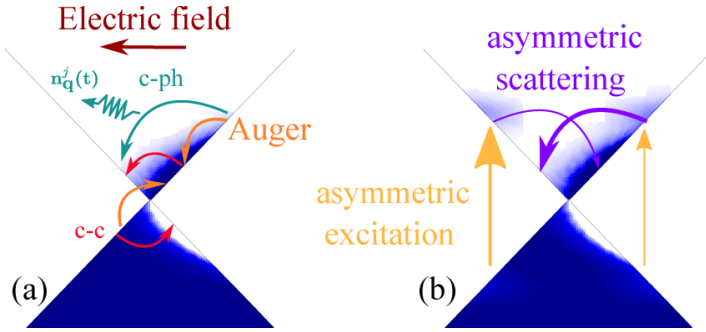

FIG. 1. Electron acceleration and scattering in an electrical field. Visualization of calculated electron distributions (blue areas) in response to an in-plane electric field (a) before and (b) after an optical excitation. Electrons become accelerated in the direction opposite to the applied field. Carrier-carrier (c-c, red arrows) including Auger processes (orange arrows) and carrier-phonon scattering (c-ph, green arrows) counteract this field-induced acceleration until a quasiequilibrium electron distribution is reached. Then, an optical excitation (yellow arrows) creates a nonequilibrium distribution. The excitation is asymmetric with respect to the Dirac point, since the initial quasiequilibrium distribution is shifted and asymmetrically broadened in the opposite direction of the field. As a result, the optical excitation is weakened on this side of the Dirac point due to the increased Pauli blocking. For the same reason, many-particle scattering becomes also strongly asymmetric, reducing the initial asymmetry of the quasiequilibrium electron distribution.

equations of motion for the electron occupation probability $\rho_{\mathbf{k}}^{\lambda}=\left\langle a_{\mathbf{k} \lambda}^{+} a_{\mathbf{k} \lambda}\right\rangle$, the microscopic polarization $p_{\mathbf{k}}=\left\langle a_{\mathbf{k} v}^{+} a_{\mathbf{k} c}\right\rangle$, and the phonon number $n_{\mathbf{q}}^{j}=\left\langle b_{\mathbf{q} j}^{+} b_{\mathbf{q} j}\right\rangle$. Here, we have introduced $a_{\mathbf{k} \lambda}^{+}, a_{\mathbf{k} \lambda}\left(b_{\mathbf{q} j}^{+}, b_{\mathbf{q} j}\right)$ as creation and annihilation operators of electrons (phonons) in the valence or conduction band $\lambda=v, c$ (phonon mode $j$ ) and the momentum $\mathbf{k}(\mathbf{q})$. The derivation of the equations requires knowledge of the many-particle Hamilton operator $H$. In this work, we take into account the (i) free carrier and phonon contribution $H_{0}$, (ii) carrier-carrier $H_{\mathrm{c}-\mathrm{c}}$ and (iii) carrier-phonon $H_{\mathrm{c}-\mathrm{ph}}$ interaction accounting for Coulomb- and phonon-induced scattering, (iv) the carrier light coupling $H_{\mathrm{c}-1}$ that is treated on a semiclassical level, and (v) the interaction of an external electric field $\mathbf{E}$ with electrons $H_{\mathrm{c}-\mathrm{f}}$. The latter is given by

$$
H_{\mathrm{c}-\mathrm{f}}=-i e_{0} \mathbf{E} \cdot \sum_{\mathbf{k}} a_{\mathbf{k} \lambda}^{\dagger} \nabla_{\mathbf{k}} a_{\mathbf{k} \lambda},
$$

with the elementary charge $e_{0}$ and the electric field $\mathbf{E}$. Exploiting the Heisenberg equation, we derive graphene Bloch equations in the second-order Born-Markov approximation,

$$
\begin{gathered}
\dot{\rho}_{\mathbf{k}}^{\lambda}=\Gamma_{\mathbf{k} \lambda}^{\text {in }}\left(1-\rho_{\mathbf{k}}^{\lambda}\right)-\Gamma_{\mathbf{k} \lambda}^{\text {out }} \rho_{\mathbf{k}}^{\lambda} \\
\pm 2 \operatorname{Im}\left(\Omega_{\mathbf{k}}^{v c, *} p_{\mathbf{k}}\right)-\frac{e_{0}}{\hbar} \mathbf{E} \cdot \nabla_{\mathbf{k}} \rho_{\mathbf{k}}^{\lambda}, \\
\dot{p}_{\mathbf{k}}=i \Delta \omega_{\mathbf{k}} p_{\mathbf{k}}-i \Omega_{\mathbf{k}}^{v c}\left(\rho_{\mathbf{k}}^{c}-\rho_{\mathbf{k}}^{v}\right)-\frac{e_{0}}{\hbar} \mathbf{E} \cdot \nabla_{\mathbf{k}} p_{\mathbf{k}}, \\
\dot{n}_{\mathbf{q}}^{j}=\Gamma_{\mathbf{q} j}^{\mathrm{em}}\left(n_{\mathbf{q}}^{j}+1\right)-\Gamma_{\mathbf{q} j}^{\mathrm{abs}} n_{\mathbf{q}}^{j}-\gamma_{\mathrm{ph}}\left(n_{\mathbf{q}}^{j}-n_{\mathbf{q}, \mathrm{B}}^{j}\right) .
\end{gathered}
$$

It is a coupled system of differential equations describing the dynamics of the time- and momentum-resolved electron occupation probability $\rho_{\mathbf{k}}^{\lambda}(t)$, the microscopic polarization $p_{\mathbf{k}}(t)$, and the phonon number $n_{\mathbf{q}}^{j}(t)$. The dynamics of electrons in the conduction and the valence band is symmetric in the considered undoped graphene samples. The appearing Rabi frequency is defined as $\Omega_{\mathbf{k}}^{v c}(t)=i \frac{e_{0}}{m_{0}} \mathbf{M}_{\mathbf{k}}^{v c} \cdot \mathbf{A}(t)$ with the free-electron mass $m_{0}$, the vector potential $\mathbf{A}(t)$, and the optical matrix element $\mathbf{M}_{\mathbf{k}}^{v c}=\left\langle\mathbf{k} v\left|\nabla_{\mathbf{k}}\right| \mathbf{k} c\right\rangle$. The $+(-)$ sign stands for $\lambda=c(\lambda=v)$. Furthermore, we have introduced $\hbar \Delta \omega_{\boldsymbol{k}}(t)=\left[\varepsilon_{\boldsymbol{k}}^{v}-\varepsilon_{\boldsymbol{k}}^{c}+i \gamma_{\mathbf{k}}(t)\right]$ with the electronic dispersion $\varepsilon_{\mathbf{k}}^{\lambda}$ and the dephasing rate $\gamma_{\mathbf{k}}(t)$. The time- and momentumdependent dephasing $\gamma_{\mathbf{k}}(t)$ and in- and out-scattering rates $\Gamma_{\mathbf{k} \lambda}^{\text {in/out }}(t)$ include carrier-carrier and carrier-phonon scattering channels. The dynamics of the phonon number $n_{\mathbf{q}}^{j}$ is driven by the emission and absorption rates $\Gamma_{\mathbf{q} j}^{\mathrm{em} / \mathrm{abs}}(t)[31,32], n_{\mathbf{q}, \mathrm{B}}^{j}$ is the initial Bose distribution for the phonon number, and $\gamma_{\mathrm{ph}}$ is the experimentally determined phonon decay rate [33]. For more details on the many-particle scattering and dephasing rates, cf. Refs. [31,32,34].

Transforming the system to a moving reference frame via the coordinate transformation $\mathbf{k} \rightarrow \mathbf{k}-\frac{e_{0}}{\hbar} \mathbf{E} t$ and $\frac{d}{d t} \rightarrow$ $\frac{d}{d t}-\frac{e_{0}}{\hbar} \mathbf{E} \cdot \nabla_{\mathbf{k}}$, the field terms in the Bloch equations (2)(4) disappear [35]. Then, these equations correspond to the standard graphene Bloch equations without electric field, where the dynamics induced by the field is hidden in the motion of the coordinates. Therefore, applying an electric field induces a shift of $\rho_{\mathbf{k}}^{\lambda}$ and $p_{\mathbf{k}}$ in $\mathbf{k}$ space. Numerically solving the Bloch equations gives full microscopic access to the timeand energy resolved carrier and phonon dynamics under the influence of an electric field.

For two-dimensional materials, the current density is defined as $\mathbf{j}(t)=-\frac{g e_{0}}{m_{0} A} \sum_{i f}\left\langle f\left|\mathbf{p}-e_{0} \mathbf{A}\right| i\right\rangle\left\langle a_{f}^{+} a_{i}\right\rangle$ with initial and final states $i, f$, the sample area $A$, the momentum $\mathbf{p}=-i \hbar \nabla_{\mathbf{r}}$, and the degeneracy factor $g$, which equals 8 in graphene when spin and valley degeneracy as well as electron-hole symmetry are taken into account. Since we are interested in transport properties, we consider only the intraband current density together with a homogeneity assumption, such that state $i$ equals state $f$. The occurring quantity $\left\langle f\left|\nabla_{\mathbf{r}}\right| f\right\rangle=\frac{i m_{0}}{\hbar^{2}} \nabla_{\mathbf{k}} \varepsilon_{\mathbf{k}}^{c}$ can then be determined by solving the Schrödinger equation for the Bloch functions $|f\rangle$ yielding the current density [36]

$$
\mathbf{j}(t)=-\frac{g e_{0}}{\hbar A} \sum_{\mathbf{k}} \rho_{\mathbf{k}}^{c}(t) \nabla_{\mathbf{k}} \varepsilon_{\mathbf{k}}^{c}
$$

In conventional materials, the electronic dispersion is parabolic, i.e., $\varepsilon_{\mathbf{k}}^{c}=\hbar^{2} \mathbf{k}^{2} /(2 m)$, and the current density is proportional to the momentum $\mathbf{k}$, such that the current diverges with time in the case without many-particle scattering. The linear band structure of graphene $\varepsilon_{\mathbf{k}}^{c}=\hbar v_{\mathrm{F}}|\mathbf{k}|$ with the Fermi velocity $v_{\mathrm{F}}$ leads to a qualitatively different behavior of the current density. The latter is now proportional to the unity vector $\nabla_{\mathbf{k}}|\mathbf{k}|=\mathbf{e}_{\mathbf{k}}=\mathbf{k} /|\mathbf{k}|$. As a result, it is not affected by the magnitude of the momentum but solely by its direction, and thus in contrast to conventional materials the generation and decay of the current density can be influenced by both carrierphonon and carrier-carrier scattering [37]. Field-induced shift of the carrier occupation in the linear band structure induces an acceleration of carriers up to the constant Fermi velocity, and it results, in the case of no scattering, in a finite saturation current for large times. The focus of this work lies in the photocurrent 
density that reads in graphene

$$
\mathbf{j}^{\mathrm{ph}}(t)=\mathbf{j}(t)-\mathbf{j}_{0}=-\frac{g e_{0} v_{\mathrm{F}}}{A} \sum_{\mathbf{k}} \rho_{\mathbf{k}}^{\mathrm{ph}}(t) \mathbf{e}_{\mathbf{k}},
$$

with the stationary current $\mathbf{j}_{0}$ before the optical excitation and the photoinduced occupation change $\rho_{\mathbf{k}}^{\mathrm{ph}}(t)=\rho_{\mathbf{k}}(t)-\rho_{\mathbf{k}}^{0}$.

\section{CARRIER DYNAMICS}

The crucial ingredient for understanding the generation and decay of the photocurrent is the microscopic understanding of the dynamics of optically excited carriers $\rho_{\mathbf{k}}(t)$. First, we calculate the quasiequilibrium carrier distribution $\rho_{\mathbf{k}}^{0}$ and a dark current $j_{0}$ in an electric field by solving the graphene Bloch equations [Eqs. (2)-(4)] without optical excitation. This situation has been thoroughly investigated in Ref. [27]. Once a stationary distribution is reached, we optically excite the system and study its optical response in terms of the generation and decay of a photocurrent determined by the spectral distribution of the electron occupation $\rho_{\mathbf{k}}(t)$; cf. Eq. (6).

The microscopic background of the photocurrent generation, defined as the current change in response to an optical excitation, is illustrated in Fig. 2 for different electric field strengths. To gain a better understanding of the underlying carrier dynamics, we first study snapshots of the carrier distribution $\rho_{k_{x}}\left(t_{0}\right)$ at the fixed time of $0.2 \mathrm{ps}$ after the excitation pulse [Fig. 2(a)]. The carrier distributions resemble the stationary distribution $\rho_{k_{x}}^{0}$, which are similar to Fermi distributions that are shifted and distorted along the field direction. The impact of optically excited carriers can be seen in Fig. 2(b), where the photoinduced occupation change $\rho_{k_{x}}^{\mathrm{ph}}=\rho_{k_{x}}-\rho_{k_{x}}^{0}$ is plotted along the direction of the applied field. Since the initial carrier distribution is shifted to the positive side of the Dirac cone due to the presence of an electric field, the Pauli blocking is stronger on this side such that asymmetric optical excitation and many-particle scattering occurs (Fig. 1). This causes a larger peak in $\rho_{k_{x}}^{\text {ph }}$ on the left side of the Dirac point, and this peak height difference decreases with the field. A measure for the photocurrent density is the asymmetry of the photoinduced carrier distribution $\Delta \rho_{\mathbf{k}}^{\mathrm{ph}}=$ $\rho_{|\mathbf{k}|}^{\mathrm{ph}}-\rho_{-|\mathbf{k}|}^{\mathrm{ph}}$ that is plotted in Fig. 2(c) for the exemplary field of $0.32 \mathrm{~V} \mu \mathrm{m}^{-1}$. The blue (red) area corresponds to the region in momentum space where the asymmetry is positive (negative), i.e., more carriers are found on the right (left) side of the Dirac point with respect to the quasiequilibrium distribution without the optical excitation. Although the blue region is spectrally broader, the amplitude of the red area is much larger, resulting in an overall negative photocurrent density, as shown in Fig. 2(d). Note that the negative photocurrent density stems from asymmetries in both the scattering toward the Dirac point and the optical excitation. However, the ultrafast carrier-carrier and carrier-phonon processes dominate over the asymmetric excitation in most situations.

A stronger electrical field induces a larger shift of the carrier distribution in momentum space and enhances the asymmetry of the entire distribution with respect to the Dirac point. Although this induces an enhancement of the asymmetric optical excitation and many-particle scattering, the photocurrent actually increases due to the stronger impact
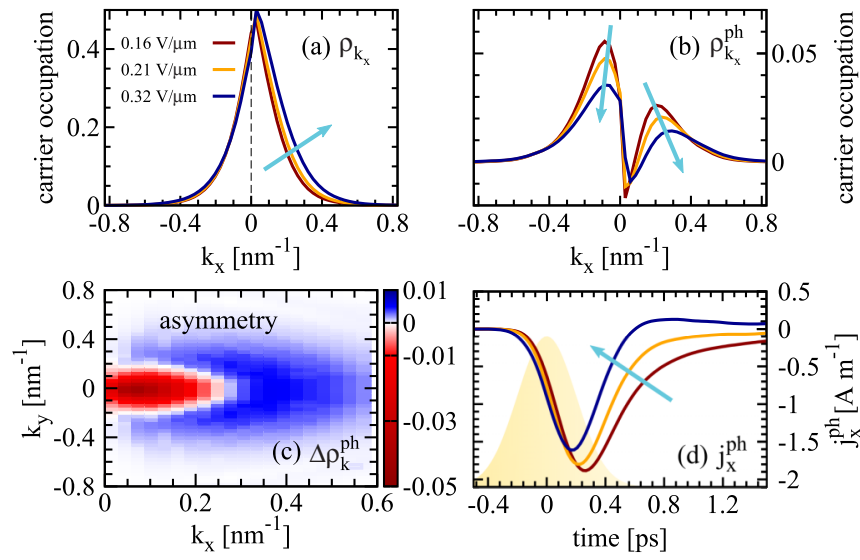

FIG. 2. Microscopic view on photocurrent generation. (a) Snapshot of the carrier distribution $\rho_{k_{x}}$ at the fixed time of 0.2 ps after the excitation pulse (linearly polarized into the $y$ direction, centered at an energy of $600 \mathrm{meV}$ corresponding to a carrier momentum of $0.5 \mathrm{~nm}^{-1}$ ) for three different electrical fields. (b) Photoinduced occupation change $\rho_{k_{x}}^{\mathrm{ph}}=\rho_{k_{x}}-\rho_{k_{x}}^{0}$ in the field direction. (c) To illustrate the impact of the excitation on the current generation, the asymmetry of the distribution is quantified by $\Delta \rho_{\mathbf{k}}^{\mathrm{ph}}=\rho_{|\mathbf{k}|}^{\mathrm{ph}}-\rho_{-|\mathbf{k}|}^{\mathrm{ph}}$ for the exemplary field $0.32 \mathrm{~V} \mathrm{\mu m}^{-1}$. (d) Resulting photocurrent density $j_{k_{x}}^{\text {ph }}$ for different electric fields with the excitation pulse in the background.

of the field-induced shift of optically generated charge carriers. This leads to a less pronounced negative amplitude with increasing electric fields [Fig. 2(a)]. Approximately 0.4 ps after the optical excitation, we observe for the strongest applied field that the photocurrent density even becomes positive; cf. the blue line in Fig. 2(d). Again, this is due to the faster acceleration of carriers in comparison to the backscattering toward the Dirac point, which results in more carriers at the right side of the Dirac cone and a positive asymmetry in the carrier distribution [in analogy to Fig. 2(c)], giving rise to a positive photocurrent density.

In a simplified picture, the generation and decay of a photocurrent density in graphene in the presence of an electric field can be understood as a four-step process: (i) The direct response stems from the asymmetric optical excitation giving a negative contribution to the photocurrent density. This effect is important for small excitation energies and large temperatures, where the Pauli blocking on the right side of the Dirac cone strongly reduces the optical excitation on this side. (ii) The many-particle scattering bringing charge carriers to the vicinity of the Dirac point is also asymmetric due to the initial asymmetric carrier distribution in the presence of an electric field. This effect induces a negative contribution to the photocurrent and is the dominant mechanism for the investigations performed at room temperature in Fig. 2. (iii) The optically excited charge carriers are shifted by the electric field. This results in a positive photocurrent contribution and dominates the photocurrent density when the many-particle scattering is suppressed via strong screening (e.g., in high-dielectric substrates) or Pauli blocking (e.g., at high temperatures). (iv) Finally, the photocurrent density decays within a few picoseconds, which is caused by carrier-phonon and carrier-carrier scattering bringing the system back to its quasiequilibrium carrier 
distribution in the electric field. The negative and positive contributions to the photocurrent density build up on a time scale given by the pulse duration and decay within a few picoseconds, suggesting the possibility of ultrafast photodetection.

\section{PHOTOCURRENT DEPENDENCE ON TEMPERATURE AND SUBSTRATE}

Having understood the microscopic mechanisms that govern the photocurrent, we now investigate how it can be controlled by external experimentally accessible quantities, such as temperature and substrate. Our calculations reveal that at enhanced temperatures, the amplitude of the photocurrent density becomes smaller; cf. Fig. 3(a). Increasing the temperature corresponds to a much broader distribution with a less pronounced asymmetry in the stationary carrier distribution $\rho_{k}^{0}$ before the optical excitation [Fig. 3(b)]. Hence, the photocurrent density, which mainly results from asymmetric scattering, becomes smaller. At very large temperatures, we observe the emergence of a positive photocurrent density component approximately $0.2 \mathrm{ps}$ after the optical excitation [blue line in Fig. 3(a)]. Analogous to the field dependence, the sign of the photocurrent density is determined by the interplay between field-induced acceleration of the optically excited carriers away from the Dirac point and counteracting many-particle scattering processes bringing the carriers toward the Dirac point.

To study this in detail, we define rates $\Gamma_{i}=\left.\frac{d}{d t} j\right|_{i} \propto$ $\left.\dot{\rho}_{\boldsymbol{k}}\right|_{i}=\frac{i}{\hbar}\left[H_{i}, \rho_{\boldsymbol{k}}\right]$ with $i=\mathrm{f}, \mathrm{c}-\mathrm{c}, \mathrm{c}-\mathrm{ph}$ allowing us to distinguish between the field (f), carrier-carrier (c-c), and carrier-phonon (c-ph) contribution to the temporal evolution of the carrier density. According to Eq. (6), the photocurrent is determined by the underlying carrier distribution $\rho_{\boldsymbol{k}}(t)$. The latter is found by solving the Bloch equations (2)-(4), where the different contributions enter additively and can thus be distinguished without switching off individual processes. Furthermore, the resulting contribution to the photocurrent is defined as $j_{i}=$ $\int d t \Gamma_{i}$. In Fig. 3(c) the photocurrent is split into two parts, $j^{\text {ph }}=j_{\mathrm{f}}+j_{\mathrm{s}}$, where $j_{\mathrm{f}}$ describes the direct influence of the electric field (in the presence of the other contributions) to the photocurrent density, i.e., the shifting of optically excited charge carriers generating a positive photocurrent density. The second part $j_{\mathrm{s}}=j_{\mathrm{c}-\mathrm{c}}+j_{\mathrm{c}-\mathrm{ph}}$ includes carrier-carrier and carrier-phonon scattering acting against the electric field and inducing a negative contribution to the photocurrent.

At high temperatures, the field contribution $j_{\mathrm{f}}$ becomes dominant and gives rise to the observed positive photocurrent. While the initial rise of $j_{\mathrm{f}}$ and the underlying field rate $\Gamma_{\mathrm{f}}$ remains nearly unchanged with increasing temperatures, the decrease of the scattering rates $\Gamma_{\mathrm{s}}$ in the first 0.2 ps due to higher symmetry in the carrier occupation and therefore weaker Pauli blocking is crucial [Fig. 3(d)]. Since carrier-carrier scattering is more sensitive to Pauli blocking (two electronic scattering processes), the rate decrease with increasing $T$ is more pronounced than in the case of carrier-phonon scattering. In fact, the carrier-phonon rate almost stays constant, since the phonon occupation becomes larger at higher $T$, compensating for the general reduction of the scattering efficiency to a large extent. Note that scattering with K-TO phonons is crucial here, since the strength of the corresponding matrix element is the largest
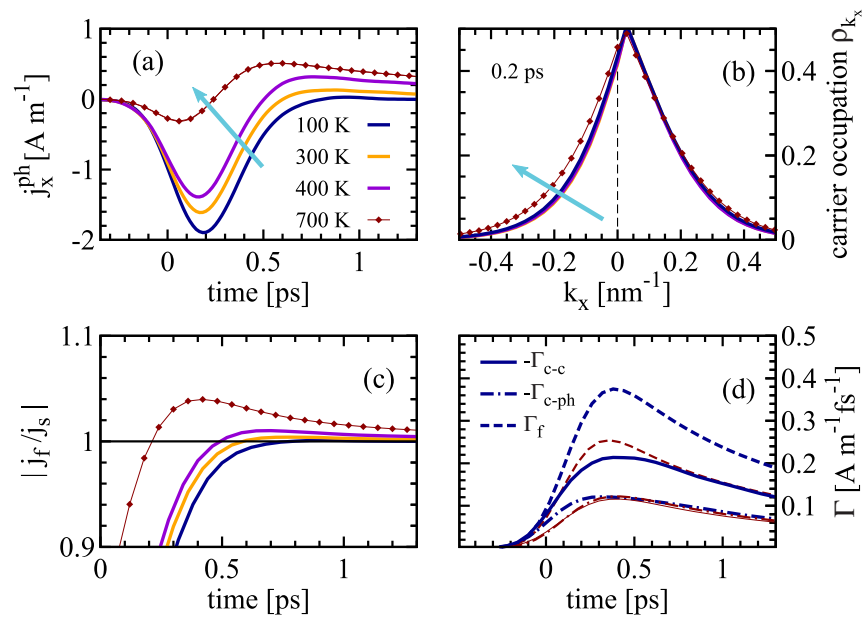

FIG. 3. Temperature study. (a) Photocurrent density $j_{x}^{\mathrm{ph}}$ for three different temperatures $T$. (b) Snapshot of the carrier distribution $\rho_{k_{x}}$ at the fixed time of $0.2 \mathrm{ps}$ after the excitation pulse illustrating a reduction of the asymmetry with respect to the Dirac point at large $T$. Since the difference of the carrier distribution is very small in the range between 100 and $400 \mathrm{~K}$, we also show the extremely high temperature of $700 \mathrm{~K}$. (c) The ratio of the field- $\left(j_{\mathrm{f}}\right)$ and scattering-induced $\left(j_{\mathrm{s}}\right)$ photocurrent density determines the sign of the photocurrent. At higher $T$, the field contribution becomes stronger than the scattering contribution, resulting in a positive current. (d) Temporal evolution of the field rate $\Gamma_{f}$ as well as the carrier-carrier $\Gamma_{\mathrm{c}-\mathrm{c}}$ and carrier-phonon $\Gamma_{\mathrm{c}-\mathrm{ph}}$ scattering rate contributing to the generation of the photocurrent density for two different temperatures. The broader carrier distribution at higher $T$ gives rise to a stronger Pauli blocking and therefore considerably less efficient carrier-carrier $\Gamma_{\mathrm{c}-\mathrm{c}}$ scattering.

and since its angular dependence favors backscattering across the Dirac cone having the largest impact on the asymmetry of the carrier distribution [32]. Having in mind that scattering induces the negative photocurrent contribution in Fig. 3(a) via carrier-phonon (green arrows in Fig. 1) and carrier-carrier (red arrows in Fig. 1) scattering across the Dirac cone, the reduced many-particle scattering explains the suppression of the negative photocurrent contribution and the eventual emergence of a positive current as the temperature increases.

As we have seen so far, carrier-carrier scattering has a strong impact on the photocurrent density in graphene. It is thus interesting to investigate how the photocurrent density changes for different substrates, since the Coulomb scattering strength can be controlled via the substrate-induced dielectric screening. A higher dielectric constant of the substrate corresponds to a stronger background screening and a weaker carrier-carrier scattering. In this work, the substrate is assumed to be only on one side of graphene. Here, we introduce an averaged dielectric background constant $\varepsilon_{\mathrm{bg}}=\frac{1}{2}\left(\varepsilon_{\mathrm{s}}+1\right)$, where $\varepsilon_{\mathrm{s}}$ is the static screening constant of the substrate (the standard substrate is $\mathrm{SiC}$ ) and 1 describes the dielectric constant of air. The screening is determined by the static Lindhard formula [30]:

$$
\varepsilon(\mathbf{q}, t)=1-2 \frac{V_{\mathbf{q}}}{\varepsilon_{\mathrm{bg}}} \sum_{\mathbf{k}, \lambda \lambda^{\prime}} \frac{\rho_{\mathbf{k}}^{\lambda}(t)-\rho_{\mathbf{k}-\mathbf{q}}^{\lambda^{\prime}}(t)}{\varepsilon_{\mathbf{k}}^{\lambda}-\varepsilon_{\mathbf{k}-\mathbf{q}}^{\lambda^{\prime}}}\left|C_{\mathbf{q}}^{\lambda \lambda^{\prime}}(\mathbf{k})\right|^{2},
$$



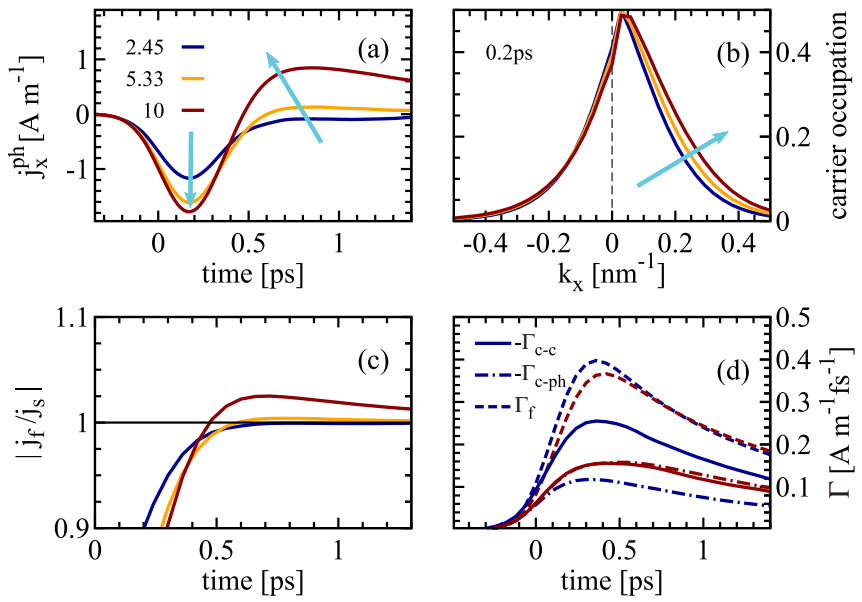

FIG. 4. Substrate study. (a) Photocurrent density $j_{x}^{\text {ph }}$ for three different substrates characterized by the dielectric background constants $\varepsilon_{\mathrm{bg}}^{\mathrm{SiO}_{2}}=2.45, \varepsilon_{\mathrm{bg}}^{\mathrm{SiC}}=5.33$, and an exemplary high-dielectric substrate with $\varepsilon_{\mathrm{bg}}=10$. (b) Snapshot of the carrier distribution $\rho_{k_{x}}$ at the fixed time of $0.2 \mathrm{ps}$ after the excitation pulse illustrating a higher asymmetry for larger $\varepsilon_{\mathrm{bg}}$. (c) The sign of the photocurrent density is determined by the interplay of the counteracting field- $\left(j_{\mathrm{f}}\right)$ and scattering-induced $\left(j_{\mathrm{s}}\right)$ contributions. We find that the field contribution exceeds the scattering contribution for large $\varepsilon_{\mathrm{bg}}$ explaining the appearance of a positive current density. (d) Temporal evolution of the underlying field rate $\Gamma_{f}$ as well as the carrier-carrier $\Gamma_{\mathrm{c}-\mathrm{c}}$ and carrier-phonon $\Gamma_{\mathrm{c}-\mathrm{ph}}$ scattering rate for two different substrates. The carrier-carrier scattering becomes considerably weaker for high dielectric substrates.

with the Coulomb potential $V_{\mathbf{q}}$ and the prefactor $C_{\mathbf{q}}^{\lambda \lambda^{\prime}}(\mathbf{k})=$ $\int d \mathbf{r} \Psi_{\lambda}^{*}(\mathbf{k}) e^{-i \mathbf{q} \cdot \mathbf{r}} \Psi_{\lambda^{\prime}}(\mathbf{k})$ with the tight-binding wave functions $\Psi_{\lambda}(\mathbf{k})$. The time dependence of the carrier distribution $\rho_{\mathbf{k}}^{\lambda}(t)$ is explicitly taken into account by solving the graphene Bloch equations.

Figure 4(a) shows the temporal evolution of the photocurrent density $j_{x}^{\text {ph }}$ for three different substrates including $\mathrm{SiO}_{2}\left(\varepsilon_{\mathrm{bg}}=2.45\right), \mathrm{SiC}\left(\varepsilon_{\mathrm{bg}}=5.33\right)$, and an exemplary highdielectric substrate $\left(\varepsilon_{\mathrm{bg}}=10\right)$. The larger $\varepsilon_{\mathrm{bg}}$ is, the stronger is the substrate-induced screening of the Coulomb interaction and the larger is the asymmetry in the carrier occupation $\rho_{k_{x}}$ [Fig. 4(b)], resulting in a more pronounced negative amplitude of the photocurrent density. For substrates with $\varepsilon_{\mathrm{bg}}>5$, we observe the appearance of a positive photocurrent density component approximately $0.5 \mathrm{ps}$ after the optical excitationsimilarly to the observation at high temperatures in Fig. 3(a). This behavior is due to the counteracting contributions of the electric field and the many-particle scattering. The sign change is directly mirrored by the ratio of the field- $\left(j_{\mathrm{f}}\right)$ and scatteringinduced $\left(j_{\mathrm{s}}\right)$ photocurrent density contribution [Fig. 4(c)]. We find that $j_{\mathrm{f}}$ exceeds $j_{\mathrm{s}}$ for large $\varepsilon_{\mathrm{bg}}$ approximately $0.5 \mathrm{ps}$ after the optical excitation, explaining the positive current density in Fig. 4(a).

While carrier-phonon scattering becomes more efficient for large $\varepsilon_{\mathrm{bg}}$ due to a higher asymmetry in the carrier occupation, the carrier-carrier scattering is generally strongly reduced through the substrate-induced screening, resulting in a considerable weakening of the scattering contribution $j_{\mathrm{s}}$. In particular, the Coulomb-induced asymmetric scattering across the Dirac cone (red arrows in Fig. 1) is strongly suppressed, explaining the significant decrease of $\Gamma_{\mathrm{c}-\mathrm{c}}$ in Fig. 4(d). Additionally, Auger processes are also reduced, leading to a smaller field rate (due to a smaller carrier density) especially during the duration of the optical excitation, such that the scattering dominates up to $0.5 \mathrm{ps}$. This is expressed in Fig. 4(c) by small ratios of $j_{\mathrm{f}}$ to $j_{\mathrm{s}}$ for large dielectric constants. Moreover, carrier-phonon scattering, which competes with carrier-carrier scattering, is enhanced. In total, the reduction of the field rate and the increased phonon scattering explain the more pronounced negative part of the photocurrent density for high dielectric constants.

\section{TUNING THE PHOTOCURRENT}

The amplitude of the photocurrent density determines the responsivity of a photodetector, while its rise and fall time determine the detector bandwidth. Since the origin of the negative photocurrent is a result of an interplay between the field-induced acceleration and many-particle scattering, its rise is not exponential and therefore it is difficult to define a rise time. However, we find that the temporal position of the negative amplitude of the photocurrent density is independent of temperature and substrate, since the behavior is governed by asymmetric scattering. For higher substrates the asymmetry is larger, but the scattering strength is reduced. The same holds for increasing the temperature. The position of the minimum can be tuned with the field, which is acting against the asymmetric scattering by accelerating carriers away from the Dirac point. The strength of the electric field defines the time window for asymmetric scattering, i.e., a stronger field induces a smaller time window resulting in an earlier appearance of the minimum.

The description of the decay time of the photocurrent is also complicated due to the presence of a zero crossing under certain conditions. Starting from negative photocurrent densities, field and relaxation processes compete with the asymmetric scattering and determine the decay. When the photocurrent density becomes positive, relaxation processes and asymmetric scattering lead to a decay. Overall, the photocurrent density decays faster for negative $(\sim 2 \mathrm{ps})$ than for positive current contributions ( $\sim 8 \mathrm{ps}$ ), reflecting the difference between scattering- and field-dominated regions. In the first case, the field supports the decay of the negative photocurrent, while it acts against the relaxation processes and asymmetric scattering in the second case.

The amplitude of the photocurrent and therewith the responsivity of the photodetector (operating in the photoconductive regime) can be tuned with the electric field $E$, substrate $\varepsilon_{\mathrm{bg}}$, and temperature $T$; cf. Fig. 5. The largest amplitude can be reached at relatively small $E$ [Fig. 5(a)], relatively large $\varepsilon_{\text {bg }}$ [Fig. 5(b)], and low $T$ [Fig. 5(c)]. The higher the temperature, the broader is the carrier distribution and the weaker is the asymmetric scattering, resulting in a smaller negative amplitude. For large substrate-induced dielectric screening, the carrier-carrier scattering is strongly suppressed, resulting in a more asymmetric carrier distribution, enhanced asymmetric carrier-phonon scattering, and larger (initial) current amplitudes. At later times $(>0.5 \mathrm{ps})$, the field-induced contribution to the current becomes dominant 


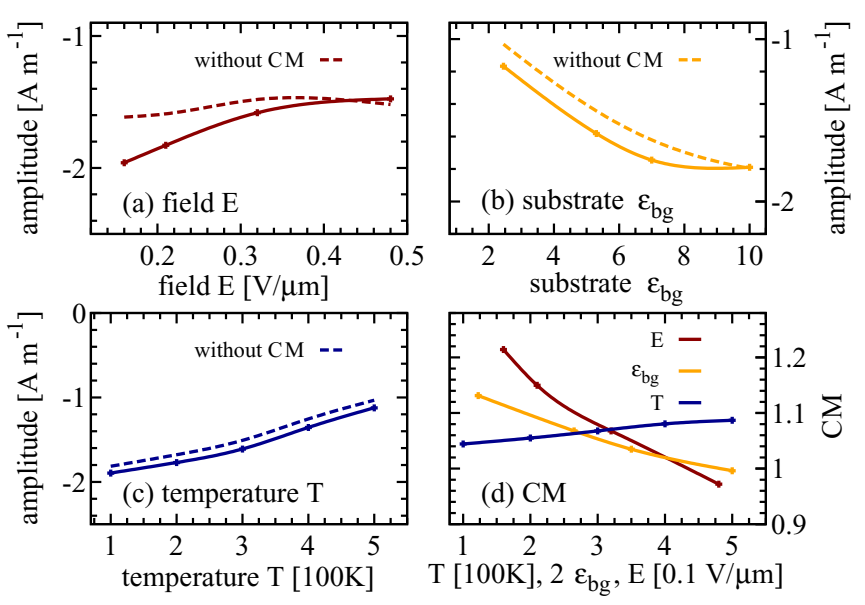

FIG. 5. Tuning the photocurrent amplitude. The photocurrent amplitude and the photodetector responsivity can be controlled as a function of (a) electrical field $E$, (b) substrate $\varepsilon_{\mathrm{bg}}$, and (c) temperature $T$. The dashed lines show the behavior without taking into account the enhancement of the carrier density through Auger scattering. The largest amplitude can be reached at low $T$, relatively small $E$, and relatively large $\varepsilon_{\mathrm{bg}}$. (d) Impact of carrier multiplication (CM) in dependence of $E, \varepsilon_{\mathrm{bg}}$, and $T$.

and gives rise to a positive amplitude (not shown) for large $\varepsilon_{\mathrm{bg}}$. Finally, smaller electrical fields allow a larger time window for asymmetric scattering enhancing the current amplitude.

Finally, we discuss the impact of carrier multiplication (CM) on the photocurrent in graphene. The photoconduction effect relies on an increased conductivity due to a photoenhanced carrier density. Here, Auger scattering processes are expected to play an important role, since they can increase the carrier density via carrier multiplication and thereby are expected to have a positive effect on the photocurrent density; cf. Fig. 1(a). The carrier multiplication is defined as $\mathrm{CM}(t)=$ $\frac{n(t)-n_{0}}{n_{\text {opt }}(t)}$ with the stationary carrier density before the optical excitation $n_{0}$, the optically induced carrier density $n_{\text {opt }}(t)$, and the total carrier density including the optical excitation as well as all scattering processes $n(t)$. CM takes place, i.e., $\mathrm{CM}>1$, when the impact excitation exceeds the inverse process of Auger recombination resulting in an increased carrier density and an enhanced field-induced contribution to the photocurrent density. We find this situation for relatively small electrical field strengths [Fig. 5(d)]. The CM decreases with the field and drops below 1 for $E \approx 0.5 \mathrm{~V} \mu \mathrm{m}^{-1}$. The reason for this behavior is the spectrally broader stationary carrier distribution $\rho_{\mathbf{k}}^{0}$ at larger fields [Fig. 2(a)] resulting in an enhanced Pauli blocking of Auger processes. A larger CM supports a stronger field rate $\Gamma_{\mathrm{f}}$, since more carriers can be shifted. As a result, we would expect the photocurrent to become positive or at least the negative component to be reduced at lower electrical field strength. However, at low $E$, the field rate $\Gamma_{\mathrm{f}}$ also becomes slower, opening a larger time window for asymmetric scattering, which enhances the counteracting scattering rates $\Gamma_{\mathrm{c}-\mathrm{c}}$ and $\Gamma_{\mathrm{c}-\mathrm{ph}}$. As a result, we find an even larger negative amplitude for smaller $E$ in spite of the stronger CM; cf. the solid versus dashed line in Fig. 5(a) directly comparing the case with and without the impact of CM, respectively.

The dependence of the negative photocurrent amplitude on substrate and temperature basically follows the behavior of the CM [Fig. 5(d)]. Increasing the dielectric screening leads to a weaker Coulomb-induced scattering resulting in less $\mathrm{CM}$, such that the difference between the amplitude of the photocurrent density with (solid) and without CM (dashed) vanishes for high substrates [Fig. 5(b)]. CM is also tunable via temperature, since the initial stationary carrier density before optical excitation is temperature-dependent. The latter becomes larger at lower temperatures due to field-induced dark carrier multiplication [27], providing larger Pauli blocking. As a result, the conventional CM, i.e., multiplication of optically excited carriers, becomes larger at enhanced temperatures.

In summary, we provide a microscopic view on the generation of photocurrent in intrinsic graphene in an in-plane electric field. Treating the time- and momentum-resolved interplay of field-induced acceleration of optically excited carriers and carrier-carrier and carrier-phonon scattering on the same microscopic footing, we reveal the microscopic foundation of the photocurrent generation in graphene based on the photoconduction effect. In particular, we discuss the impact of Auger-induced carrier multiplication, and we demonstrate the tunability of the responsivity of graphene-based photodetectors with experimentally accessible quantities, such as electric field, temperature, and substrate.

\section{ACKNOWLEDGMENTS}

This project has received funding from the European Union's Horizon 2020 research and innovation programme under Grant Agreement No. 696656 (Graphene Flagship). Furthermore, we acknowledge support from the Swedish Research Council (VR). The computations were performed on resources at Chalmers Centre for Computational Science and Engineering (C3SE) provided by the Swedish National Infrastructure for Computing (SNIC). Finally, we thank Andreas Knorr (TU Berlin) for fruitful discussions.
[1] F. H. L. Koppens, T. Mueller, P. Avouris, A. C. Ferrari, M. S. Vitiello, and M. Polini, Nat. Nano 9, 780 (2014).

[2] Z. Sun and H. Chang, ACS Nano 8, 4133 (2014).

[3] M. Buscema, J. O. Island, D. J. Groenendijk, S. I. Blanter, G. A. Steele, H. S. J. van der Zant, and A. Castellanos-Gomez, Chem. Soc. Rev. 44, 3691 (2015).

[4] E. J. H. Lee, K. Balasubramanian, R. T. Weitz, M. Burghard, and K. Kern, Nat. Nano 3, 486 (2008).
[5] J. Park, Y. H. Ahn, and C. Ruiz-Vargas, Nano Lett. 9, 1742 (2009).

[6] F. Xia, T. Mueller, R. Golizadeh-Mojarad, M. Freitag, Y.-M. Lin, J. Tsang, V. Perebeinos, and P. Avouris, Nano Lett. 9, 1039 (2009).

[7] F. Xia, T. Mueller, Y.-M. Lin, A. Valdes-Garcia, and P. Avouris, Nat. Nano 4, 839 (2009).

[8] T. Mueller, F. Xia, and P. Avouris, Nat. Photon. 4, 297 (2010). 
[9] X. Xu, N. M. Gabor, J. S. Alden, A. M. van der Zande, and P. L. McEuen, Nano Lett. 10, 562 (2010).

[10] N. M. Gabor, J. C. W. Song, Q. Ma, N. L. Nair, T. Taychatanapat, K. Watanabe, T. Taniguchi, L. S. Levitov, and P. Jarillo-Herrero, Science 334, 648 (2011).

[11] M. Freitag, T. Low, and P. Avouris, Nano Lett. 13, 1644 (2013).

[12] T. J. Echtermeyer, P. S. Nene, M. Trushin, R. V. Gorbachev, A. L. Eiden, S. Milana, Z. Sun, J. Schliemann, E. Lidorikis, K. S. Novoselov, and A. C. Ferrari, Nano Lett. 14, 3733 (2014).

[13] M. Freitag, T. Low, F. Xia, and P. Avouris, Nat. Photon. 7, 53 (2013).

[14] M. Mittendorff, S. Winnerl, J. Kamann, J. Eroms, D. Weiss, H. Schneider, and M. Helm, Appl. Phys. Lett. 103, 021113 (2013).

[15] M. Mittendorff, J. Kamann, J. Eroms, D. Weiss, C. Drexler, S. D. Ganichev, J. Kerbusch, A. Erbe, R. J. Suess, T. E. Murphy, S. Chatterjee, K. Kolata, J. Ohser, J. C. König-Otto, H. Schneider, M. Helm, and S. Winnerl, Opt. Express 23, 28728 (2015).

[16] S. Schuler, D. Schall, D. Neumaier, L. Dobusch, O. Bethge, B. Schwarz, M. Krall, and T. Mueller, Nano Lett. 16, 7107 (2016).

[17] G. Konstantatos, M. Badioli, L. Gaudreau, J. Osmond, M. Bernechea, F. P. G. de Arquer, F. Gatti, and F. H. L. Koppens, Nat. Nano 7, 363 (2012).

[18] C.-H. Liu, Y.-C. Chang, T. B. Norris, and Z. Zhong, Nat. Nano 9, 273 (2014).

[19] T. Winzer, A. Knorr, and E. Malic, Nano Lett. 10, 4839 (2010).

[20] D. Brida, A. Tomadin, C. Manzoni, Y. J. Kim, A. Lombardo, S. Milana, R. R. Nair, K. S. Novoselov, A. C. Ferrari, G. Cerullo, and M. Polini, Nat. Commun. 4, 1987 (2013).

[21] F. Wendler, A. Knorr, and E. Malic, Nat. Commun. 5, 3703 (2014).

[22] T. Winzer and E. Malic, Phys. Rev. B 85, 241404 (2012).

[23] F. Kadi, T. Winzer, A. Knorr, and E. Malic, Sci. Rep. 5, 16841 (2015).
[24] T. Plötzing, T. Winzer, E. Malic, D. Neumaier, A. Knorr, and H. Kurz, Nano Lett. 14, 5371 (2014).

[25] M. Mittendorff, F. Wendler, E. Malic, A. Knorr, M. Orlita, M. Potemski, C. Berger, W. A. de Heer, H. Schneider, M. Helm, and S. Winnerl, Nat. Phys. 11, 75 (2015).

[26] I. Gierz, M. Mitrano, J. C. Petersen, C. Cacho, I. C. E. Turcu, E. Springate, A. Stöhr, A. Köhler, U. Starke, and A. Cavalleri, J. Phys.: Condens. Matter 27, 164204 (2015).

[27] R. Jago, F. Wendler, and E. Malic, 2D Mater. 4, 021031 (2017).

[28] A. Junck, G. Refael, and F. von Oppen, Phys. Rev. B 90, 245110 (2014).

[29] M. Kira and S. Koch, Prog. Quantum Electron. 30, 155 (2006).

[30] H. Haug and S. W. Koch, Quantum Theory of the Optical and Electronic Properties of Semiconductors (World Scientific, Singapore, 2009).

[31] E. Malic and A. Knorr, Graphene and Carbon Nanotubes: Ultrafast Optics and Relaxation Dynamics (Wiley-VCH, New York, 2013).

[32] E. Malic, T. Winzer, E. Bobkin, and A. Knorr, Phys. Rev. B 84, 205406 (2011).

[33] K. Kang, D. Abdula, D. G. Cahill, and M. Shim, Phys. Rev. B 81, 165405 (2010).

[34] F. Kadi, T. Winzer, E. Malic, A. Knorr, F. Göttfert, M. Mittendorff, S. Winnerl, and M. Helm, Phys. Rev. Lett. 113, 035502 (2014).

[35] T. Meier, G. von Plessen, P. Thomas, and S. W. Koch, Phys. Rev. Lett. 73, 902 (1994).

[36] G. Czycholl, Theoretische Festkörperphysik (Springer, Berlin, 2008).

[37] D. Sun, C. Divin, M. Mihnev, T. Winzer, E. Malic, A. Knorr, J. E. Sipe, C. Berger, W. A. de Heer, P. N. First, and T. B. Norris, New J. Phys. 14, 105012 (2012). 